\title{
Computational Fluid Dynamics Optimization of an Extraoral Vacuum Aerosol Cup for Airborne Disease Control in Dental Offices
}

\author{
Peter Liu ${ }^{1}$ (D)
}

Received: 20 July 2021 / Revised: 30 September 2021 / Accepted: 6 October 2021 / Published online: 28 October 2021

(C) Institute of Earth Environment, Chinese Academy Sciences 2021

\begin{abstract}
Droplet and aerosol transmission of COVID-19 are the most important concerns in dental clinics, due to the generation of large amounts of infected aerosol and droplets mixed with patient's saliva during the procedures. The current approach to prevent airborne disease transmission is an extraoral aerosol suction unit: a stand-alone vacuum module with a segmented arm and cup. Despite the need for disease control in dental offices, these units are rarely seen due to the loud noise produced by vacuum, bulky size, and high cost. This paper describes the aerodynamic design optimization of an affordable, 3D printable, Extraoral Vacuum Aerosol Cup (EVAC) that can be directly connected to existing standard 7/16" central vacuum highvolume evacuator (HVE) valves used for intraoral saliva absorption in a dental office. These HVEs are typically unsuitable for extraoral suction due to their low vacuum force. However, they can be used for extraoral suction, if the cup attachment is aerodynamically optimized for maximum suction efficiency. Fifteen different designs of EVAC are proposed and their suction processes were simulated with computational fluid dynamics. Droplets of various sizes are released to mimic the droplets produced during dental operation. The suction performances of EVACs with different sizes and shapes were compared to find out the designs with optimal performance. Prototypes of the optimized EVAC are 3D printed and tested at a dental office. Development and manufacturing of such a device will largely reduce the COVID-19 infection risk, thus improving the safety protection for both patients and doctors at dental offices.
\end{abstract}

\section{Graphic abstract}
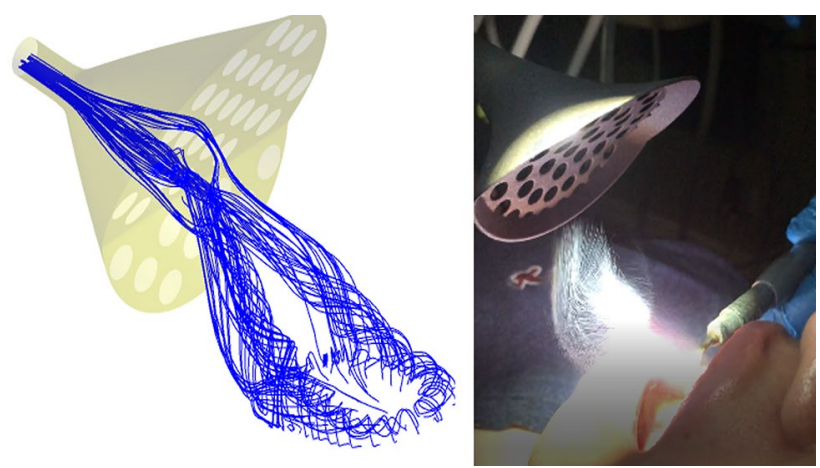

Keywords COVID-19 · Dental disease control · Aerosol suction · Extraoral suction · Computational fluid dynamics (CFD)

Peter Liu

peterliu.0909@gmail.com

1 Upper Dublin High School, Fort Washington, PA 19034,

USA 


\section{Introduction}

Dental patients and healthcare providers can be exposed to pathogenic viruses and bacteria that infect the oral cavity and respiratory tract. The dental care environment invariably carries the risk of COVID-19 infection due to face-toface communication with patients, and frequent exposure to saliva and other body fluids. Airborne spread of SARSCoV-2 through many dental procedures is well reported in literature (Ehtezazi et al. 2021; Polednik 2014). Droplet and aerosol transmission of COVID-19 are the most important concerns in dental clinics and hospitals, i.e., generation of large amounts of aerosol and droplet mixed with patient's saliva/blood during dental infected patient's cough and breathing, high-speed dental handpiece with gas or running water (Holliday et al. 2021; Matys and Grzech-Leśniak 2020). These droplets and aerosols are small enough to stay airborne for an extended period before they settle on environmental surfaces or enter the respiratory tract. Thus, the COVID-19 has high risk of spreading through droplets and aerosols from infected individuals in dental clinics and hospitals (Clarkson et al. 2020; Estrich et al. 2020).

Currently, technology for extraoral aerosol suction is the Extraoral Dental Suction System (Ehtezazi et al. 2021; Pérez et al. 2021), which removes droplets and aerosols produced during dental treatments. Extraoral Dental Suction Systems are typically rectangular vacuum modules with a 2-segmented arm for adjustment. At the end of the segmented arm is a simple acrylic pyramid/hemisphere-shaped cup for droplet capture. These devices are commonly sold by businesses such as Henry Schein Dental and Sino Dental Group. However, these systems are very expensive (most cost above $\$ 1000$ ) and are not efficient. Extraoral Dental Suction Systems use individual vacuum modules, making them noisy, hard to maneuver, and prone to damage. For these reasons, they are not commonly seen in dental offices. The current dental spittoon is designed for saliva/spit suction, which is not suitable for extraoral aerosol suction. As shown in Fig. 1, due to the urgent need, some dentists use DIY suction cups made from regular paper cups, but these DIY cups have poor durability and poor aerodynamic suction performance.

We propose to develop a new extraoral suction device that uses the built-in high-volume excavation (HVE) vacuum that is already present in dental offices. To account for the low vacuum pressure of the HVE, such a device needs to be aerodynamically optimized using data tested at a dental office and computational fluid dynamics.

There are a few dental aerosol suction devices such as (Oral appliance for removing aerosols produced during dentistry. 1992) an oral appliance that removes aerosols produced during dental operations. The apparatus includes a collector connected to a vacuum source that provides a barrier around the mouth of a patient to trap aerosols. The device is ring-shaped to surround the mouth and to allow the dentist to see into the mouth. While such a device offers an effective solution to reducing the spread of aerosols, the collector must be disposed of and replaced after every use. This creates the need to purchase many collectors, making the process expensive, and also produces unnecessary waste, making the product costly and detrimental to the environment. We would like to design an autoclavable and reusable device. It will be made of polypropylene, which is a lightweight material but can withstand heavy loads and pressures, making our device long-lasting and durable. Another device (Intraoral Device and Patent 2011) uses an external vacuum that connects to the entrance of the patient's mouth, which makes it unsuitable for absorbing airborne particles, leading to the same problems that the current systems have. Though there are other devices and patents that are designed for aerosol suction in dental offices, they are either large bulky vacuum modules that generate their own vacuum pressure (results in a loud noise output) or require attachments to be disposed of after every use. We plan to use an existing vacuum module, as an inexpensive option for dental offices to solve the problem of disease transmission. The device does not generate its own vacuum pressure but uses that of pre-existing technology (HVAC) already present in dental offices. It is an extraoral device, which means it does not touch the mouth. Thus, it does not require extra safety precautions.
Fig. 1 Current extraoral suction devices in market (Pérez et al. 2021; Extra-oral suction unit launched 2020). Left: Extraoral Dental Oral Suction Unit; middle: dry oral cup and its usage for saliva spit; right: DIY paper suction cup
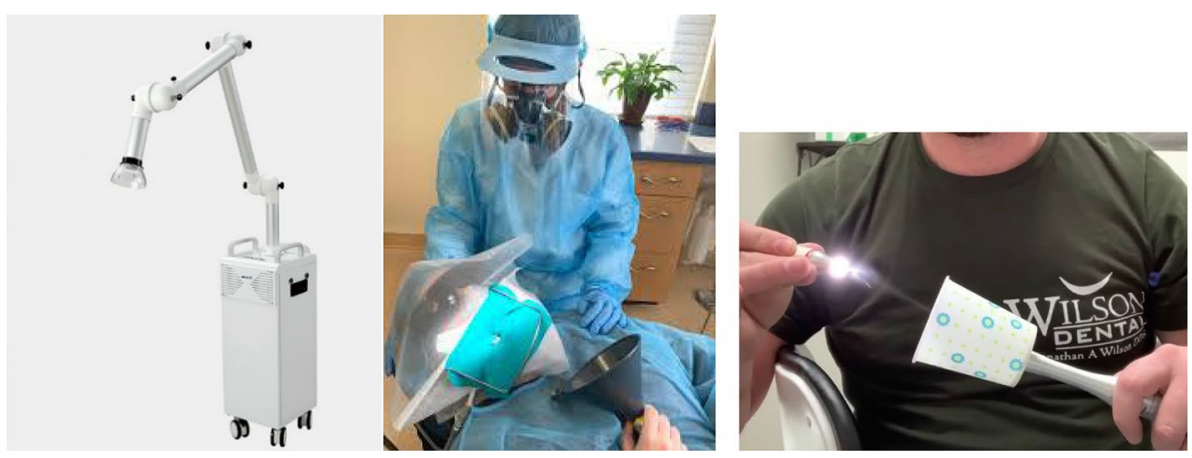
Our solution is to develop an affordable, 3D printable, and aerodynamically optimized Extraoral Vacuum Aerosol Cup (EVAC) that can be directly connected to the existing 7/16" central vacuum standard high-volume evacuator (HVE) valves in the typical dental office. Through computational fluid dynamics simulation, we will optimize the shape and size of the device for the low vacuum pressure of the HVAC. EVAC showed suction efficiency similar to or better than other patents and devices that are being sold at a much higher size and price.

\section{Methods and Analysis}

The overall concept of the suction cup is described in Fig. 2. Multiple specific designs of the device are created using CAD. Computational fluid dynamics simulation will be performed to optimize the designed cup shape with consideration of suction vacuum pressure and volume flow rate provided by central vacuum. The efficiency of the designs will be determined through CFD simulations, and the best designs are selected for prototyping and testing. Using these steps, an Extraoral Vacuum Aerosol Cup can be designed, optimized using CFD, and finally, produced for potential testing and/or usage.

We will evaluate the performance of our suction cups by criteria such as suction efficiency, ease of use, and durability. Droplets from a dental handpiece are used to visualize and evaluate the suction performance.

\section{Problem Description}

As the Extraoral Vacuum Aerosol Cup is designed to capture aerosols exiting from a patient's mouth, it is essential that the cup must have maximum suction efficiency for effective use. To create a product that is both cost-efficient and rooted in scientific and technical principles, various shapes and measurements of the overall concept were tested. For further optimization of suction efficiency, the cup was aerodynamically optimized using Computational Fluid Dynamics, which was used to select the best-fit height and width of the device.
The overall design of the cup is funnel-shaped, with the upper lip of the funnel extending further out than the lower lip to account for the angle of the patient's mouth. During a dental operation, the cup is attached to an HVE (commonly used for intraoral saliva absorption). Since the central vacuum has a low suction force, EVAC features holes spread uniformly around the frontal surface area to increase suction force and prevent larger substances from being captured. To ensure that the product is usable in an actual dental environment, a 3D-printed prototype was developed and attached to an HVE to measure the appropriate attachment width, as well as the distance from patient to device $(9.5 \mathrm{~cm}$ viewing distance from device, $1.5 \mathrm{~cm}$ diameter for the HVE connection tube). In addition to the measurements, a video (under suitable lighting conditions and appropriate safety precaution) was taken of a patient undergoing a dental procedure with and without the use of the cup attachment to validate its practical use. In addition, particle velocity of the aerosols emitted from the patient's mouth was calculated using a frame-by-frame analysis of the videotape.

The cup will be aerodynamically optimized under specific conditions with computational fluid dynamics, using measurements of the appropriate distance and emitted particle velocity. As shown in Fig. 3, seven different cup designs, with varying curvatures and shapes, were designed and modeled in the fluid simulation software ANSYS Fluent. The various designs are selected based on the shapes of suction cups of existing extraoral suction machines on the market, typical nozzle curvatures from aerodynamics, fit of human mouth geometry, and potential design variations. The suction cup in the domain is considered as a thin wall (the inner surface of the 3D cup geometry imported from Solidworks) with a non-slip boundary condition for air. The patient's mouth is modeled as an ellipse, and the droplets are modeled as water droplets with various sizes. N1, N2, N6, and N7 have mouth-like shape, while N3, N4, and N5 has ellipsoid shape. To make the in silico simulation as realistic as possible, different forces were applied onto the droplets, such as a pressure gradient, Saffman Lift Force, and Hydrodynamic drag. Furthermore, the typical pressure of a standard HVE (high-volume evacuator) of $0.07 \mathrm{MPa}$ was considered when creating the model. After initial testing, the best cup design (highest suction efficiency) will be chosen for further

Fig. 2 Left: cup as fitted on human face; middle: planned CFD simulation; right: preliminary CAD design of the Smart Extraoral Aerosol Suction Cup

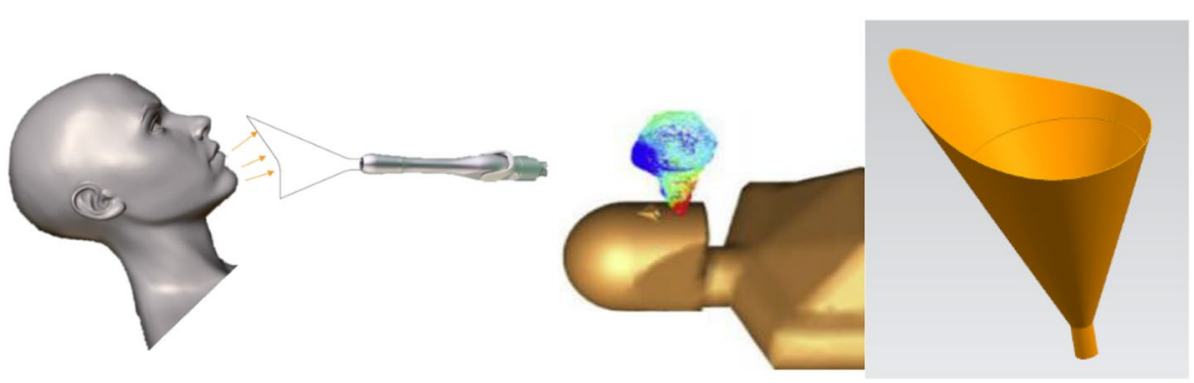


Fig. 3 Different designs for the suction cup
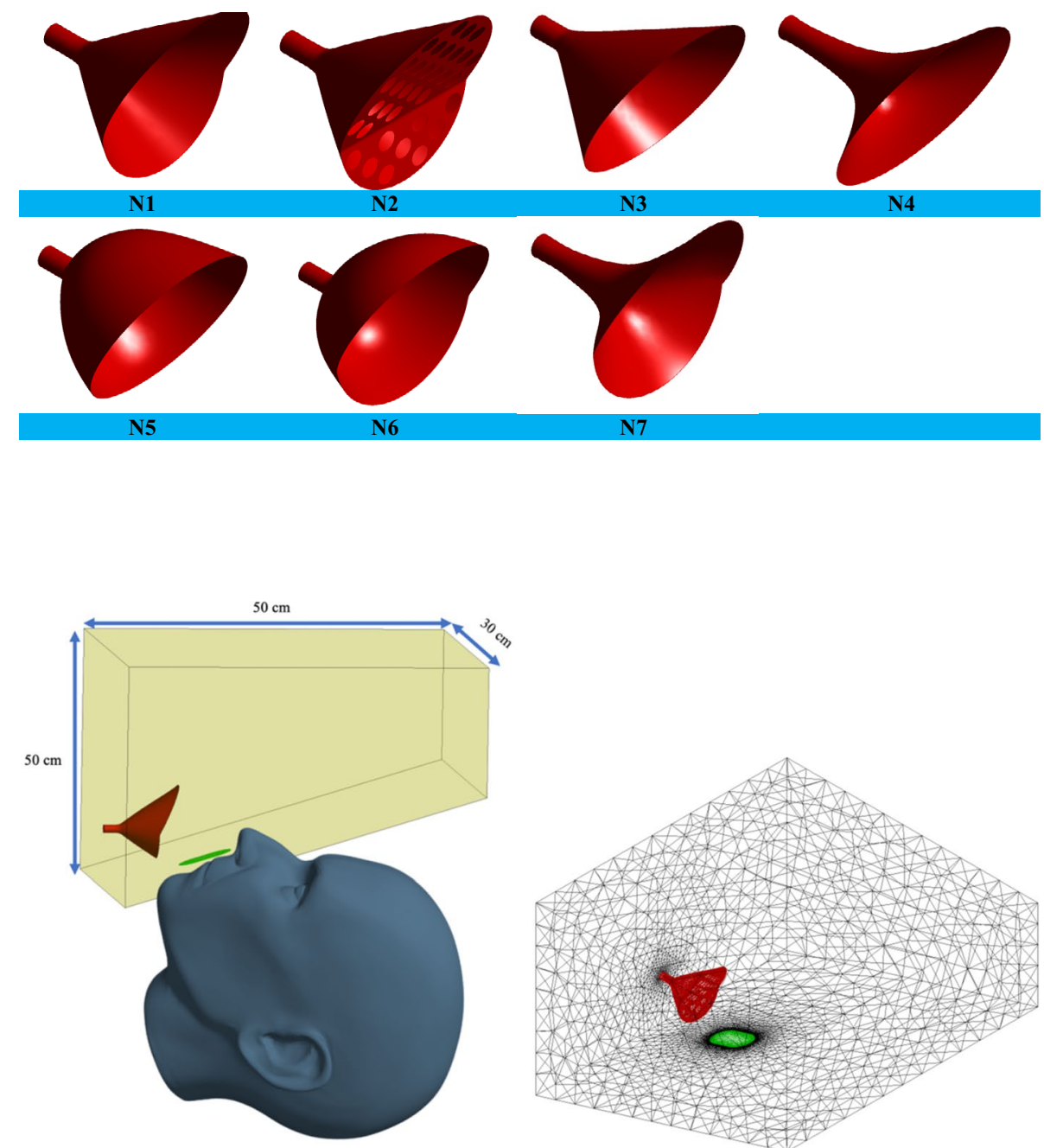

Fig. 4 Schematic view of the computational model and a typical computational grid with $1,696,910$ tetrahedron elements optimization. Various heights for the upper part and lower part as well as the cup width and suction efficiency will be tested.

The schematic view of the computational model is illustrated in Fig. 4. A typical computational grid composed of $1,696,910$ tetrahedron elements in the simulation.

In these geometries, the cup's inlet area (in front of the mouth) remains the same and equal to $7291.75 \mathrm{~mm}^{2}$. For all the cases, the cup's outlet diameter (connected to the suction pipeline) is $1.1 \mathrm{~cm}$, and the distance between cup inlet and mouth is $8 \mathrm{~cm}$. The angle between mouth and cup is $50^{\circ}$. The cup height or length is kept at $10.3 \mathrm{~cm}$. In N1, N2, N6 and N7, the cup inlet is similar to human mouth cross-section. $\mathrm{N} 2$ has some pores with $1 \mathrm{~cm}$ diameter. In N3 and N4, the inlet cross-section is the same, while the wall profile is straight and curved, respectively. In N7, everything is similar to N4, but the inlet cross-section is similar to the mouth. The only difference between N5 and N6 is the shape of inlet cross-section:
N5 is simple and N6 is mouth-like. In all the simulations, the mouth is designed as an ellipsoid with $7.6 \mathrm{~cm}$ as the major diameter and $4.4 \mathrm{~cm}$ as the minor diameter.

The droplet velocity is measured as $0.1-0.6 \mathrm{~m} / \mathrm{s}$ through analyzing video of a person during dental operation, as shown in Fig. 5. The boundary is set as $0 \mathrm{~Pa}$ as gauge pressure and $-0.07 \mathrm{MPa}$ suction head at nozzle outlet, similar to the vacuum pressure of HVE vacuum. The material properties are listed in Table 1.

\section{Numerical Procedure}

ANSYS Fluent is used to perform the numerical procedure on a structured computational grid. In the simulation, the air and liquid-water particles leave the mouth at $0.1 \mathrm{~m} / \mathrm{s}$ while the intake pressure at the nozzle outlet (smaller area) is set at $-0.07 \mathrm{MPa}$. The air flow from nostrils is not considered. As shown in later section, all air streamlines 
Fig. 5 Measurement of droplet release velocity from a movie of aerosol releasing from a person's mouth during dental operation

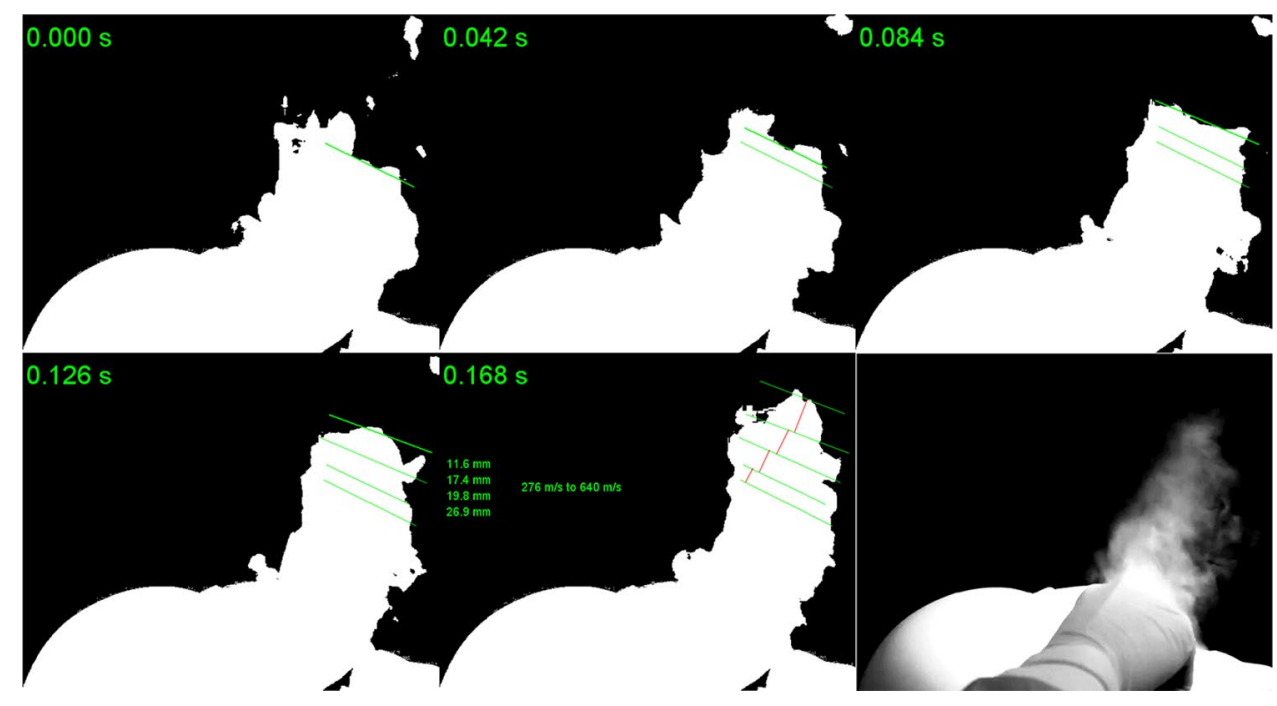

Table 1 Air and droplet properties used in this study

\begin{tabular}{lcc}
\hline & Air (primary phase) & Liquid (secondary phase) \\
\hline Viscosity $(\mathrm{kg} / \mathrm{m}-\mathrm{s})$ & $\mu_{a}=1.7894 e-05$ & $\mu_{l}=0.001003$ \\
Density $\left(\mathrm{kg} / \mathrm{m}^{3}\right)$ & $\rho_{l}=1.225 \rho_{l}=998.2$ \\
\hline
\end{tabular}

can be easily sucked in with $100 \%$ efficiency, thus air flow is not the major concern. Instead, the droplet coming out of mouth during dental operation has much higher chance of escaping from suction. The remaining boundaries of the computational box are considered as pressure outlets through which the water-liquid droplets can escape (since they are part of ambient area). Since droplets with diameters ranging from 0.5 to $10 \mu \mathrm{m}$ are prone to stay in the air for hours and transfer viruses, the mentioned range with $5 \mu \mathrm{m}$ was considered for droplet injection. The droplet sizes have normal distribution. The interaction between droplet and air is one-way and both translational and rotational motion of the droplet were modeled. The following forces were applied on the droplet: Saffman Lift Force, drag force, and pressure gradient force. Discrete Phase Model (DPM) model in Fluent is used for the two-phase model (air phase and droplet phase), where the droplet is tracked in a Lagrangian view as discrete particles. All the simulations are performed at steady state, laminar flow using SIMPLE algorithm. All the discretization was performed with second-order accuracy. The solution is considered converged when all governing residuals are less than $10^{-3}$.
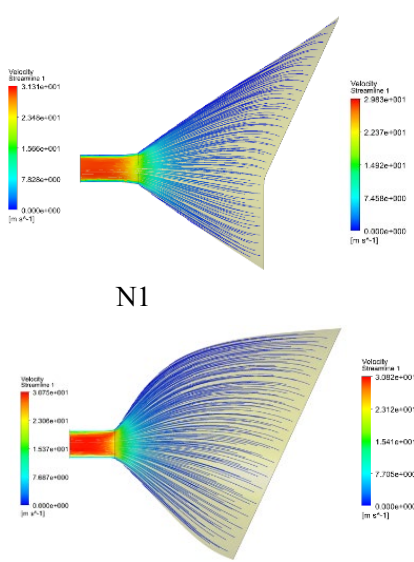

N5

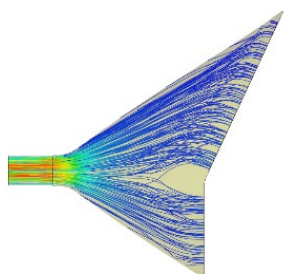

N2

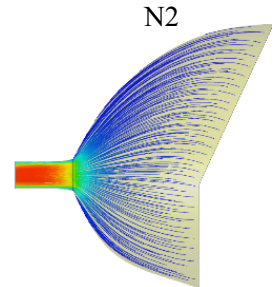

N6
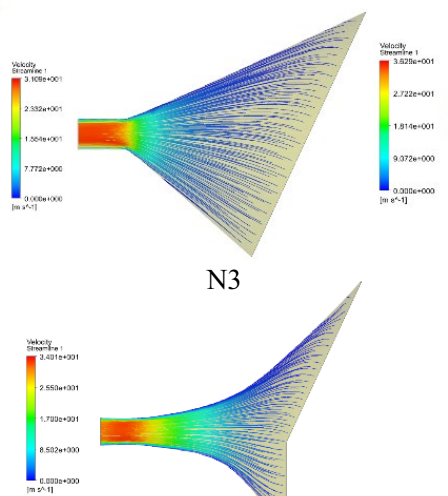

N3

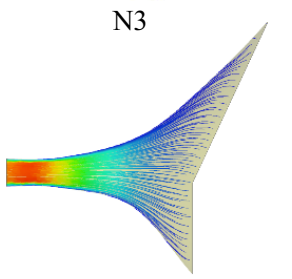

N7

Fig. 6 Fluid flow streamlines in different cups from N1 to N7 
Fig. 7 CFD simulation and streamlines of air released from mouth
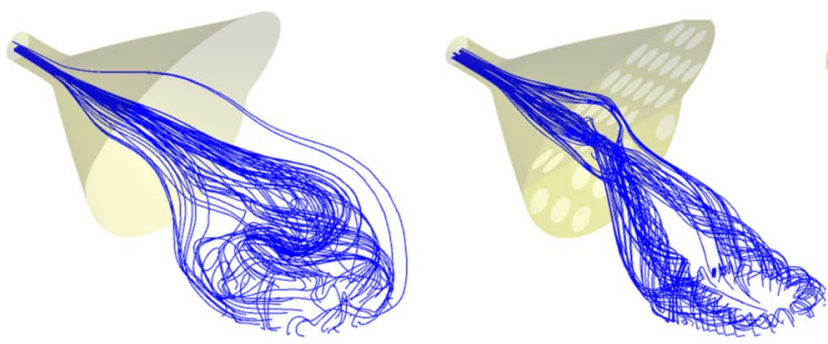

Table 2 Suction efficiency for cups of different designs

\begin{tabular}{llllllll}
\hline Case & 1 & 2 & 3 & 4 & 5 & 6 & 7 \\
\hline Efficiency & $74.84 \%$ & $75.77 \%$ & $78.54 \%$ & $81.44 \%$ & $79.02 \%$ & $75.81 \%$ & $76.07 \%$ \\
Mass flow & 0.00332 & 0.00325 & 0.00326 & 0.00353 & 0.00319 & 0.00313 & 0.00343 \\
\hline
\end{tabular}
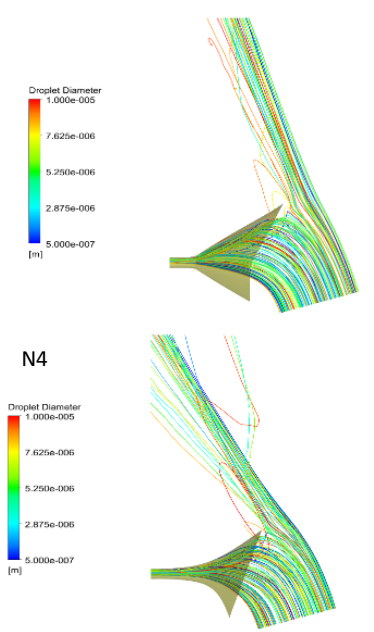
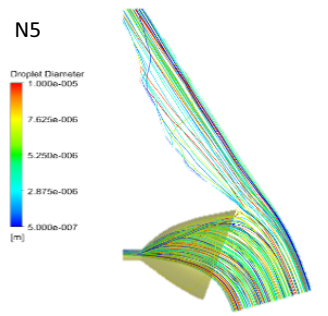
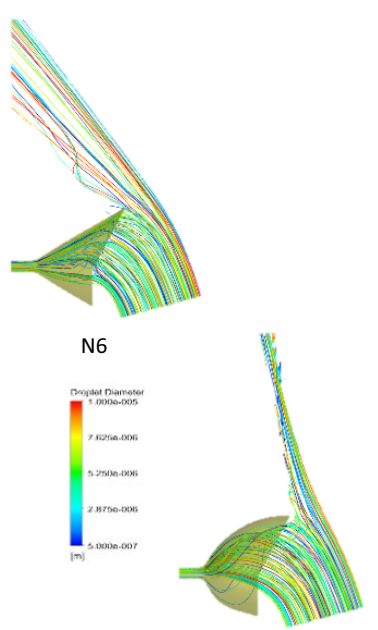
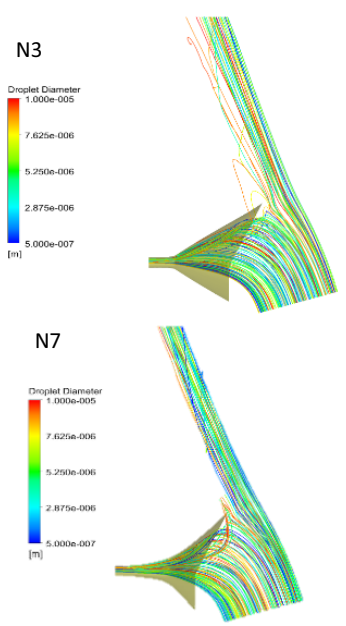

Fig. 8 CFD simulations and streamlines of droplets injected out of mouth for different cup designs

\section{Results and Discussion}

We analyze the effects of different cup designs on the suction efficiency of the cup. First, the streamline of airflow inside the cup is visualized by applying a negative suction pressure at the cup's inlet. Figure 6 shows the streamlines of aerosol droplets being sucked into nozzles of different designs. Uniform suction streamlines are found in all cup designs. As Fig. 6 shows, in mouth-like nozzles (N1, N2, N6, and N7), the air streamline can be sucked in more easily. The mouth-like nozzles also influence more areas on the mouth. In N3, despite having a uniform airflow, the affected area is less than a mouth-like nozzle.

Then, we want to check out if the suction cup can effectively siphon in the air breathed out from the mouth. To illustrate the airflow, the 100 air streamlines started from the mouth corresponding to each test case are displayed in Fig. 7. As the figure shows, all air streamlines can be easily sucked in with $100 \%$ efficiency. This means the proposed EVAC device can easily draw in all air breathing from the patient's mouth.

Next, we check out if the suction cup can effectively suck in liquid droplets injected out of the mouth. One million droplets are released from the mouth as shown in Fig. 8. The
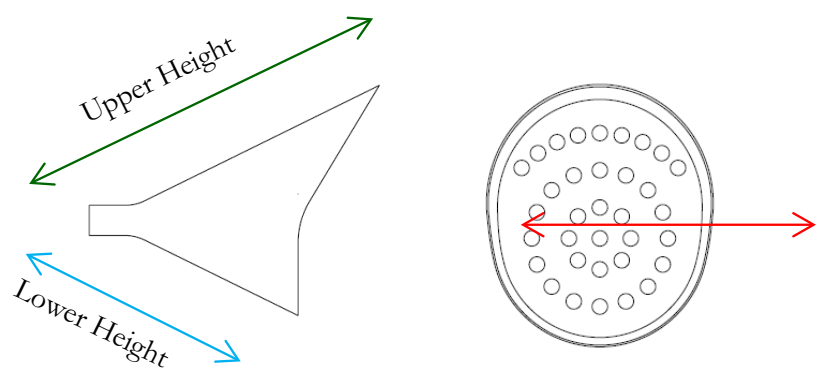

Nozzle Width

Fig. 9 Different shape factors on cup design 

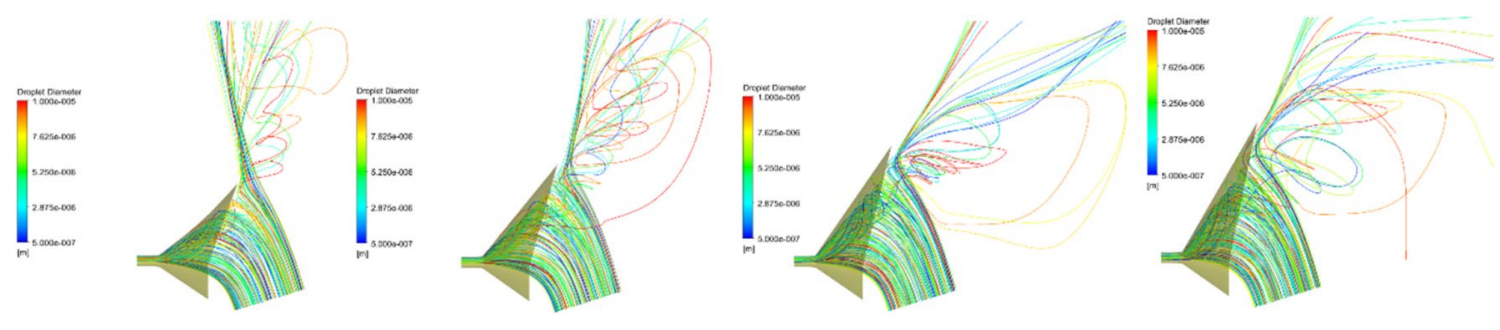

Fig. 10 CFD simulation of the original cup (control) and increase of cup top height by $20 \%, 40 \%$, and $80 \%$, respectively

Table 3 Suction efficiency for cups with different top heights

\begin{tabular}{llllll}
\hline Case & Control & $20 \%$ & $40 \%$ & $60 \%$ & $80 \%$ \\
\hline Efficiency & $74.84 \%$ & $79.77 \%$ & $84.15 \%$ & $85.90 \%$ & $84.96 \%$ \\
\hline
\end{tabular}
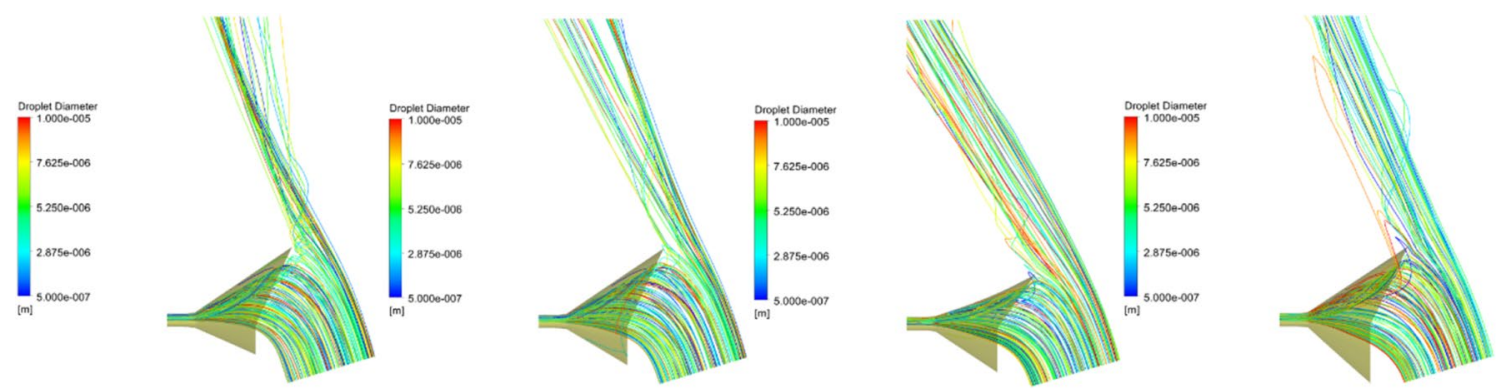

Fig. 11 CFD simulation of change of cup lower height by $-30 \%,-10 \%, 10 \%$, and $30 \%$, respectively

suction efficiency is defined as the percentage of droplets sucked into the nozzle out of the total number of droplets released. The suction efficiency and mass flow rate for different nozzles are summarized in Table. 2. N4 has the highest efficiency of $81 \%$. Making the cup's shape like a mouth decreases the efficiency although such shape leaves large operation room and is preferred by dentists while making pores on the nozzle inlet slightly enhances the efficiency. Nozzles with maximum converging area (N4 and N7) have stronger suction with higher efficiency ).

It is observed that suction efficiency does not change significantly among mouth-like nozzles considerably, so further optimizations are performed for cup N1. The upper part height, lower part height, and the cup width will be changed to test their influence on suction efficiency, as illustrated in Fig. 9.

First, the upper height of the cup is changed and tested. The upper height of the cup is increased by $20 \%, 40 \%$, and $80 \%$, respectively. The path lines of aerosol droplets for these cases are illustrated in Fig. 10, and suction efficiency is summarized in Table 3.
Table 4 Suction efficiency for cups with different lower heights

\begin{tabular}{llllll}
\hline Case & Control & $-30 \%$ & $-10 \%$ & $+10 \%$ & $+30 \%$ \\
\hline Efficiency & $74.84 \%$ & $76.31 \%$ & $75.83 \%$ & $75.11 \%$ & $73.23 \%$ \\
\hline
\end{tabular}
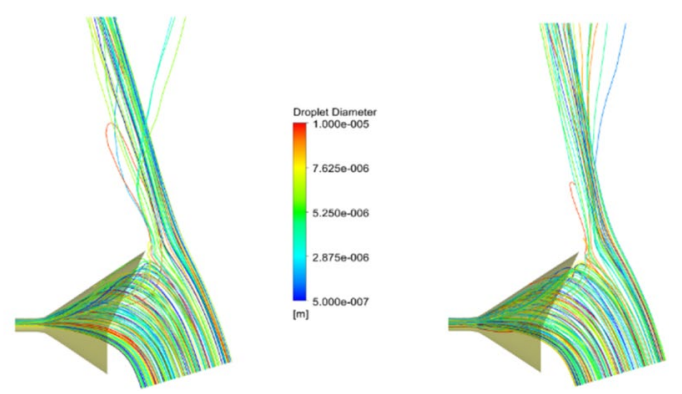

Fig. 12 CFD simulation of change of cup widths by $+20 \%$, and $+40 \%$, respectively

Increasing upper part height up to $60 \%$ increases suction efficiency from 75 to $85 \%$. Above $60 \%$, suction efficiency declines. However, increasing height too much blocks viewing area for dentists' operation. Above 40\%, suction becomes weak and particle trajectories are prone

Table 5 Suction efficiency for cups with different widths

\begin{tabular}{llll}
\hline Case & Control & $+20 \%$ & $+40 \%$ \\
\hline Efficiency & $74.84 \%$ & $75.75 \%$ & $76.42 \%$ \\
\hline
\end{tabular}


Fig. 13 Suction tests on 3D-printed prototypes
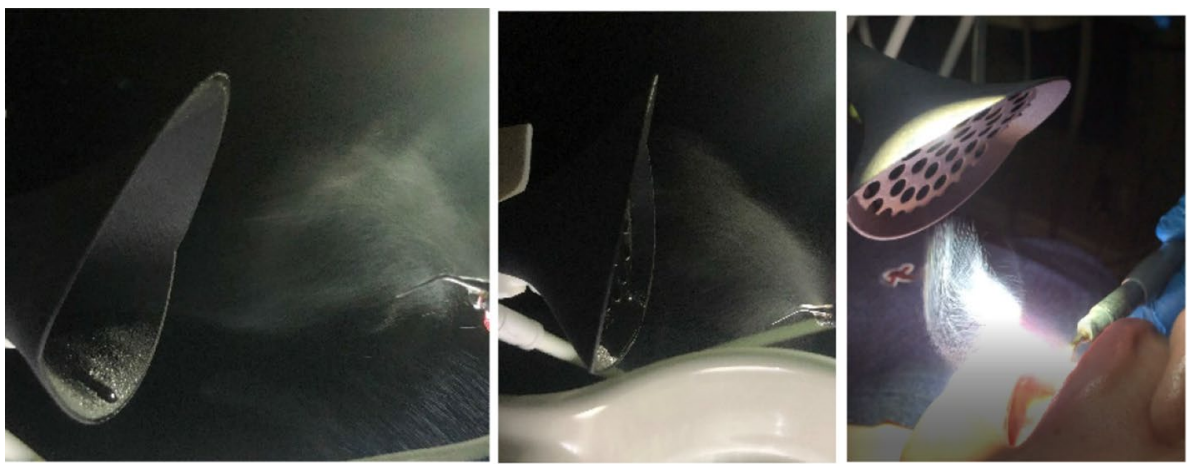

to irregular paths. Therefore, the best performance is obtained by $40 \%$ increase of upper part height.

Then, the lower height of the cup is changed and optimized. The lower height of the cup is decreased by $30 \%$ and $10 \%$, and increased by $10 \%$ and $30 \%$, respectively. The path lines of aerosol droplets for these cases are illustrated in Fig. 11, and suction efficiency is summarized in Table 4.

It is shown that changes in lower heights do not significantly influence the suction efficiency.

Finally, we studied the influence of cup width on suction performance. The suction cup widths are increased by $20 \%$ and 40\%, respectively. As shown in Fig. 12 and Table 5, increasing the width slightly increases the suction efficiency. However, increasing the width can limit dentist operation space, which is a concern for the device's practicality.

In summary, among different designs, N4 is the best design with $81 \%$ suction efficiency. Creating pores on $\mathrm{N} 1$ slightly increases the suction efficiency and can provide a mechanism to avoid suction of small parts during dental operation. By varying the upper height, lower height, and widths of the cup, the suction efficiency can improve from the initial design of $75 \%$ to $84 \%$ after optimization.

Besides suction efficiency, the performance of the suction cups should also be considered with other factors such as ease of use and durability. A few prototypes are 3D printed with Nylon material using laser sintering 3D printing. Nylon is chosen as the material for $3 \mathrm{D}$ printing because the designed cup will be able to go through the autoclave repeatedly for sterilization, and can resist a maximum heat of $130{ }^{\circ} \mathrm{C}$ with material Strength of $40 \mathrm{MPa}$ (Moradi et al. 2021). Common 3D printer materials such as PLA and ABS are not suitable to use because their glass transition temperatures are below the temperature limit for autoclaving.

The 3D-printed prototypes are tested in two ways, as shown in Fig. 13. In the first method, a water-spraying dental handpiece is placed $8 \mathrm{~cm}$ away from the suction cup. A suction efficiency of around 50\% is observed. Most of the droplets escaped from the direction opposite the suction cup. In another test with a dental handpiece inside the mouth during regular dental operation, a suction efficiency of around
$80 \%$ is observed. The higher suction efficiency is likely because the release droplets are more directional toward the suction cup when placed in the mouth compared to be freely mounted handpiece. The measured suction efficiency agrees well with the CFD simulation predictions.

\section{Conclusion}

A new extraoral suction cup is designed that can directly connect to existing HVE in dental office. CFD analysis is performed to study the droplet suction process and suction efficiency for cups of various designs. The CFD results show that proper design can reach up to $100 \%$ suction of aerosol and $80 \%$ to $84 \%$ suction efficiency of liquid droplets. The optimal design is 3D printed and tested, and a suction efficiency of $80 \%$ is achieved in the tests of regular extraoral dental operation. The proposed device offers an inexpensive, compact solution to airborne disease control and aerosol suction.

Acknowledgements The author appreciates Hai Qing of Prosthodontist at Bucks Dental Health and Esthetics for mentoring on this project and providing the facility for prototype testing and would also like to thank Mehdi Nikfar and Ratul Paul for their guidance and help on CFD simulations.

Data Availability The data that support the findings of this study are available from the author upon request.

\section{Declarations}

Conflict of interest The author declares no conflict of interests.

\section{References}

Clarkson J, Ramsay C, Richards D, Robertson C, Aceves-Martins M, Group CW, et al. (2020) Aerosol generating procedures and their mitigation in international dental guidance documents-a rapid review. Cochrane Oral Health, 1-69 
Ehtezazi T, Evans DG, Jenkinson ID, Evans PA, Vadgama VJ, Vadgama J et al (2021) SARS-CoV-2: characterisation and mitigation of risks associated with aerosol generating procedures in dental practices. Br Dent J. https://doi.org/10.1038/s41415-020-2504-8

Estrich CG, Mikkelsen M, Morrissey R, Geisinger ML, Ioannidou E, Vujicic M et al (2020) Estimating COVID-19 prevalence and infection control practices among US dentists. J Am Dent Assoc 151:815-824

Extra-oral suction unit launched (2020) BDJ Team 7:38-38

Holliday R, Allison JR, Currie CC, Edwards DC, Bowes C, Pickering K et al (2021) Evaluating contaminated dental aerosol and splatter in an open plan clinic environment: Implications for the COVID-19 pandemic. J Dent 105:103565

Hirsch et al (2011) Intraoral device. US Patent. 8075310-B2

Matys J, Grzech-Leśniak K (2020) Dental aerosol as a hazard risk for dental workers. Materials. https://doi.org/10.3390/ma13225109
Moradi M, Aminzadeh A, Rahmatabadi D, Rasouli SA (2021) Statistical and experimental analysis of process parameters of 3D nylon printed parts by fused deposition modeling: response surface modeling and optimization. J Mater Eng Perform. https://doi.org/10. 1007/s11665-021-05848-4

Oral appliance for removing aerosols produced during dentistry. US Patent. 5127411A

Pérez N, Díaz-Reissner C, Adorno C, Fretes V, Escobar J, Gamarra J et al (2021) Evaluation of the dispersion of aerosols produced during the use of the dental turbine using the ejector of the equipment and/or extraoral suction. Exploratory Study. Rev Salud Publica Parag 11:28-32

Polednik B (2014) Aerosol and bioaerosol particles in a dental office. Environ Res 134:405-409 